OPEN ACCESS

Edited by:

Dennis C. Ko,

Duke University, United States

Reviewed by:

Mireia Coscolla,

University of Valencia, Spain

Sarah Dunstan,

The University of Melbourne,

Australia

*Correspondence:

Erwin Schurr

erwin.schurr@mcgill.ca

tThese authors have contributed equally to this work

Specialty section: This article was submitted to

Evolutionary and

Population Genetics,

a section of the journal

Frontiers in Genetics

Received: 05 May 2019

Accepted: 19 August 2019 Published: 18 September 2019

Citation:

Correa-Macedo W, Cambri G and Schurr E (2019) The Interplay of Human and Mycobacterium Tuberculosis Genomic Variability.

Front. Genet. 10:865.

doi: 10.3389/fgene.2019.00865

\section{The Interplay of Human and Mycobacterium Tuberculosis Genomic Variability}

\author{
Wilian Correa-Macedo ${ }^{1,2,3 \dagger}$, Geison Cambri ${ }^{1,4 \dagger}$ and Erwin Schurr 1,2,3,5* \\ ${ }^{1}$ Program in Infectious Diseases and Immunity in Global Health, Research Institute, McGill University Health Centre, \\ Montreal, QC, Canada, ${ }^{2}$ The McGill International TB Centre, McGill University, Montreal, QC, Canada, ${ }^{3}$ Department of \\ Biochemistry, Faculty of Medicine, McGill University, Montreal, QC, Canada, ${ }^{4}$ Graduate Program in Health Sciences, School \\ of Medicine, Pontifícia Universidade Católica do Paraná, Curitiba, Brazil, ${ }^{5}$ Departments of Human Genetics and Medicine, \\ Faculty of Medicine, McGill University, Montreal, QC, Canada
}

Tuberculosis (TB), caused by the human pathogens Mycobacterium tuberculosis (Mtb) and Mycobacterium africanum, has plagued humanity for millennia and remains the deadliest infectious disease in the modern world. Mycobacterium tuberculosis and $M$. africanum can be subdivided phylogenetically into seven lineages exhibiting a low but significant degree of genomic diversity and preferential geographic distributions. Human genetic variability impacts all stages of TB pathogenesis ranging from susceptibility to infection with Mtb, progression of infection to disease, and the development of distinct clinical subtypes. The genetic study of severe childhood TB identified strong inborn singlegene errors revealing crucial pathways of vulnerability to TB. However, the identification of major TB-susceptibility genes on the population level has remained elusive. In particular, the replication of findings from candidate and genome-wide association studies across distinct human populations has proven difficult, thus hampering the characterization of reliable host molecular markers of susceptibility. Among the possible confounding factors of genetic association studies is Mtb genomic variability, which generally was not taken into account by human genetic studies. In support of this possibility, Mtb lineage was found to be a contributing factor to clinical presentation of TB and epidemiological spread of $\mathrm{Mtb}$ in exposed populations. The confluence of pathogen and human host genetic variability to TB pathogenesis led to the consideration of a possible coadaptation of $\mathrm{Mtb}$ strains and their human hosts, which should reveal itself in significant interaction effects between Mtb strain and TB-susceptibility/resistance alleles. Here, we present some of the most consistent findings of genetic susceptibility factors in human TB and review studies that point to genome-to-genome interaction between humans and Mtb lineages. The limited results available so far suggest that analyses considering joint human-Mtb genomic variability may provide improved power for the discovery of pathogenic drivers of the ongoing TB epidemic.

Keywords: tuberculosis, Mycobacterium tuberculosis complex group, host-pathogen interaction, genomic variability, gene-genotype interaction, coadaptation 


\section{INTRODUCTION}

The out-of-Africa migration exposed human populations to changing environments and henceintroduced newimmunological challenges caused by newly encountered microbial populations. The interaction with these microorganisms, especially human pathogenic species, resulted in strong selective pressures that shaped our immune system (Barreiro and Quintana-Murci, 2010; Nedelec et al., 2016; Quintana-Murci, 2019). Likewise, pathogens experienced selection via their need to overcome or avoid host defenses and to be able to spread from host to host or between host and environment. Pathogens and humans have been genetically shaped by this interplay of selective forces. Despite the incomplete mechanistic understanding of how this host-pathogen interplay impacted host and pathogen genetics, new concepts about host-pathogen coevolution have emerged (Brites and Gagneux, 2015).

An illustrative example for the above statements is provided by the adaptation of different strains of Helicobacter pylori to human populations of distinct ancestry (Kodaman et al., 2014). The coevolution of $H$. pylori, a main risk factor of human gastric cancers, and its human hosts was studied in two Colombian populations. The two populations displayed different admixture proportions of African, Amerindian, and European ancestries but presented very similar high $H$. pylori colonization levels with strikingly different incidences of gastric cancer. Helicobacter pylori isolates affecting both populations were derived from four ancestral lineages originating from Africa, Europe (2×), and Asia. Cancer incidence was found to be lowest when African ancestry of the host was matched with African ancestry of $H$. pylori. In contrast, gastric cancer risk was highest in hosts where the bacterial strain ancestry was divergent from the host ancestry (Kodaman et al., 2014). This work highlighted the importance of considering the coevolution of host and pathogen to better understand clinical progression of diseases involving infectious agents and suggested that resistance and susceptibility to infectious diseases are probably best understood when considering both the genetic make-up of the pathogen and the host. A hotly debated question in the field of tuberculosis (TB) is to what extent a similar coadaptation of Mycobacterium tuberculosis $(M t b)$, the major etiological agent of TB, and the human host has occurred. Host-centric genetics studies in TB have provided some insight in host vulnerabilities to $M t b$. However, the identification of strong genetic drivers of TB susceptibility has remained elusive (Orlova and Schurr, 2017; Abel et al., 2018; Dallmann-Sauer et al., 2018). In this review, we present selected findings from the field of host genetics of TB, describe the results of studies that implicated $M t b$ lineage as susceptibility driver, and focus on results that support a joint effect of both host and $M t b$ genomic variability on TB disease.

\section{TUBERCULOSIS DISEASE}

Human TB is caused by acid-fast bacilli belonging to the $M t b$ complex (MTBC) group, namely, Mtb and Mycobacterium africanum. The disease affects mainly the lungs, causing pulmonary TB (PTB), but it can spread to virtually any part of the body leading to distinct clinical phenotypes such as TB meningitis (TBM), a severe form of the disease characterized by progressive meningoencephalitis with necrotizing and granulomatous inflammation leading to obstruction of cerebrospinal fluid passage or infarcts of intracerebral arteries (Thwaites et al., 2013; Zumla et al., 2013). Tuberculosis is an ancestral infectious disease that accounted for an estimated 1 billion deaths in the last 200 years globally (Paulson, 2013), which is broadly consistent with the estimate of 1 billion deaths over the last 2,000 years in Europe only (Kerner et al., 2019). Tuberculosis is generally treatable, yet despite substantial efforts to control the disease, it remains the main cause of deaths by an infectious agent in the world. In 2017, the World Health Organization estimated 10 million new cases resulting in 1.57 million deaths worldwide (World Health Organization, 2018). This is partly due to the emergence of multidrug resistance including rifampicin-resistant $M t b$ for which treatment success remains low. Moreover, the TB control challenge is exacerbated by the HIV epidemic as people living with HIV are at increased risk of becoming infected by $M t b$ and of developing severe clinical forms of TB. In 2017, more than 460,000 new cases of HIVTB were detected, and approximately 300,000 deaths occurred among HIV-positive patients due to TB (World Health Organization, 2018).

\section{HUMAN GENETICS OF TB}

Several biological factors contribute to susceptibility to TB. Among these, host genetics plays a clear role in modulating risk for development of TB and its clinical forms. Early evidence for the impact of host genetics on TB susceptibility came from the observation of familial aggregation (Puffer, 1944) and a higher concordance rate of $\mathrm{TB}$ in monozygotic compared to dizygotic twins (Kallmann and Reisner, 1943). Likewise, an often-quoted example for the role of host genetics in TB risk is the Lübeck accident, which occurred in 1929 (Fox et al., 2016). Due to the accidental contamination of bacillus Calmette-Guérin (BCG) vaccines, 251 newborns received $M t b$-contaminated batches of BCG. Of the total number of vaccinated babies, 228 (90.8\%) developed diverse forms of clinical TB. A year after the accident, 77 infants had died, and 69 of these deaths were confirmed to be related to TB. Surprisingly, 23 infants (9.2\%) did not present any clinical signs for the disease, and $68 \%$ of the clinically confirmed $\mathrm{TB}$ cases progressed to spontaneous cure. These events suggested that host genetic factors played a role in TB susceptibility and severity of the disease. Interestingly, it was also shown that high levels of contamination overrode intrinsic TB resistance factors (Fox et al., 2016). These older studies along with more recent examples provide the persistent motivation for the investigation of host genetics in TB (Abel et al., 2018).

A widely accepted model for TB pathogenesis assumes two main stages of the disease process. In step 1, exposed persons become infected with $M t b$ without any clinical signs of disease. These individuals are distinguished by converting to positivity in immune assays such as the in vivo tuberculin skin test (TST) or ex vivo release of interferon $\gamma$ by blood cells in response to $M t b$ 
antigen (IGRA tests). Such persons are thought to carry Mtb bacilli and to suffer from latent TB infection (LTBI). In step 2, a subset of those who are classified as LTBI will develop TB, mostly within the first 2 years of LTBI conversion (Behr et al., 2018). In TB, natural resisters to infection have been inferred from heavy $M t b$ exposure settings in which subjects failed to convert to LTBI, and this infection resistance phenotype has been shown to have a genetic component (Orlova and Schurr, 2017; Abel et al., 2018; Simmons et al., 2018; Dallmann-Sauer et al., 2018). Studies of resistance to TST conversion have consistently implicated chromosome region 11p14, both in families from a hyperendemic area in South Africa (logarithm of the odds [LOD] score $=3.81, P=1.4 \times 10^{-5}$ ) (Cobat et al., 2009) and in a sample of ethnically mixed French families with low TB endemicity (LOD score $=3.65, P=2.08 \times$ $10^{-5}$; combined sample populations: LOD score $=4.54, P=2.4 \times$ $10^{-6}$ ) (Cobat et al., 2015). In an Ugandan population, chromosome regions $2 \mathrm{q} 21-2 \mathrm{q} 24$ and $5 \mathrm{p} 13-5 \mathrm{q} 22$ reached suggestive $P$ values $\left(P=3 \times 10^{-4}\right.$ and $5 \times 10^{-4}$, respectively) for linkage with persistent TST negativity (Stein et al., 2008), and in a Ghanaian sample, IL10 haplotypes were associated with low circulating cytokine levels, which were enriched in TST-positive versus TST-negative persons (odds ratio $[\mathrm{OR}]=2.09, P=1.7 \times 10^{-2}$ ) (Thye et al., 2009).

In $\mathrm{PTB}$, chromosome region $8 \mathrm{q} 12-\mathrm{q} 13$ was linked to disease in 96 Moroccan families ( $\mathrm{LOD}$ score $=3.49, P=3 \times 10^{-5}$ ) (Baghdadi et al., 2006), and subsequent fine mapping revealed association between PTB and a cluster of single-nucleotide variants (SNVs) in high linkage disequilibrium overlapping the TOX gene. This SNV cluster initially failed to be significantly associated with PTB in a Malagasy sample. However, stratifying the analysis for patients with age at onset of TB younger than 25 years replicated the association for rs2726600 in TOX (Grant et al., 2013). Moreover, a PTB-susceptibility locus has been detected on chromosome region 20 q13 by a genome-wide linkage study in Malawi and South African families (LOD score = 3.1, $P=8 \times 10^{-5}$ ) (Cooke et al., 2008). This linked region was further screened with 40 SNVs, and association tests using selected markers in combined samples found protective associations for MC3R $\left(\mathrm{OR}=0.67, P=4 \times 10^{-3}\right)$ and CTSZ $(\mathrm{OR}=0.49, P=9 \times$ $10^{-3}$ ) with PTB. Additional genome-wide linkage studies reached suggestive significance on chromosome 20q13 for families from Uganda (Stein et al., 2008) and Thailand (Mahasirimongkol et al., 2009). An independent study employing a South African population found association of variants present in $M C 3 R$ and CTSZ with PTB $\left(P=4 \times 10^{-4}\right.$ and $<1 \times 10^{-4}$, respectively) (Adams et al., 2011), but in an Iranian sample, only $M C 3 R$ variants were associated $\left(\mathrm{OR}=1.56, P=5 \times 10^{-3}\right)$ (Hashemi et al., 2013). Chromosome 11 also harbors evidence of genetic factors controlling PTB. A genome-wide association study (GWAS) of a Ghanaian sample found the variant rs2057178 located on region 11 p13 downstream of the WT1 gene associated with protection from TB $\left(\mathrm{OR}=0.77, P=2.63 \times 10^{-9}\right)$. This association was replicated with diminished effect size in Gambian $(\mathrm{OR}=0.8$, $\left.P=4.87 \times 10^{-4}\right)$ and Russian samples $(\mathrm{OR}=0.91, P=0.02)$ (Thye et al., 2012). Two independent GWAS replicated marker rs2057178 in two samples from South Africa $(\mathrm{OR}=0.62, P=$ $\left.2.71 \times 10^{-6}\right)($ Chimusa et al., 2014) and Morocco $(\mathrm{OR}=0.78, P=$ 0.043) (Grant et al., 2016).
To date, the HuGE navigator's phenopedia search engine lists more than 200 genes with variants that had been reported by candidate gene studies or GWAS as being associated with either susceptibility or protection to PTB. The top genes in the number of publications reporting significant genotypic associations are HLA-DRB1, VDR, and SLC11A. Variants in these genes were almost exclusively detected by candidate gene approach (Stein et al., 2017). HLA-DRB1 is the exception given that it has been recently identified in a GWAS employing a large Icelandic population of 277,643 controls and 8,162 cases (Sveinbjornsson et al., 2016). A shared aspect of genetic studies of TB susceptibility is the poor replication of risk variants across independent studies and different populations. Associations of variants in the SLC11A1 gene are among the most stable findings across different populations, although with varying effect sizes (Stein et al., 2017; Abel et al., 2018).

One of the strongest protective genetic effects for PTB detected at the population level has been described for chromosome region $5 q 33.3$ in a combined sample of Ugandan and Tanzanian HIV patients (Sobota et al., 2016). After following up HIV patients from TB hyperendemic areas, $314 \mathrm{HIV}$-infected patients remained free of $\mathrm{TB}$, whereas 267 developed active disease. Contrasting these two groups in a case-control GWAS identified the risk SNV rs4921437 located in an intron of the $U B L C P 1$ gene at $51 \mathrm{~kb}$ downstream from $I L 12 B(\mathrm{OR}=0.37$, $\left.P=2.11 \times 10^{-8}\right)$. The variant mapped to an annotated histone modification site, suggesting an impact on RNA expression levels and potentially an important role of epigenetic effects in TB susceptibility. However, no functional data for this SNV with either gene were reported (Sobota et al., 2016). The implication of $I L 12 B$ alleles as genetic vulnerability to TB is interesting due to its link to Mendelian susceptibility to mycobacterial disease (MSMD). Patients with MSMD, who are characterized by severe clinical disease caused by weakly virulent mycobacteria, often harbor deleterious mutation in genes controlling IFNG/IL12mediated immunity that can also predispose to TB (Abel et al., 2018). While individual mutations in MSMD genes are rare, it has been shown that a collection of loss of function mutations in MSMD genes can explain a substantial proportion of TB cases (Alcais et al., 2005). Another example for an important role of loss of function mutations in genes of the IFNG/IL12/ IL23 circuitry is provided by TYK2 deficiency. Homozygosity for the common TYK2 P1104A allele is a strong risk factor for TB explaining an estimated 1\% of TB deaths in Europe (BoissonDupuis et al., 2018; Kerner et al., 2019). Interestingly, the data suggested a strong selective pressure against the susceptibility allele since the frequency of P1104A in Europeans has decreased from $9 \%$ to $4.2 \%$ over the last 4,000 years (Boisson-Dupuis et al., 2018). Given its very strong impact on TB risk, the P1104A allele does provide a good opportunity to test for an interaction with $M t b$ strains and possible coadaptation.

An unexpected outcome of genetic studies trying to pinpoint TB-susceptibility genes was the limited success in the identification of common (minor allele frequency $>1 \%$ ) alleles as genetic risk factors for TB susceptibility. It is possible that genetic heterogeneity resulting from the combination of different common and rare DNA variants may give rise to a complex model 
of inheritance in which candidate gene or GWAS approaches fail to capture major genetic susceptibility drivers (Abel et al., 2018; Dallmann-Sauer et al., 2018). In this respect, it remains to be seen whether the application of whole genome or exome sequencing including the approach of focusing on extreme phenotypes and less common variants with strong effect, such as described for TYK2, can provide further insights. Perhaps, an equally important issue is phenotype definition. For example, the clinical definition of PTB may encompass distinct disease stages that are under distinct genetic control as has been shown in a mouse model of mycobacterial infection (Di Pietrantonio et al., 2010). Aside from host-centric characteristics, the genetic variability of the $M t b$ strain infecting subsets of subjects within a sample may partially account for the low success of genetic studies, from the low effect findings to poor marker consistency between different populations. Mounting experimental evidence suggests that MTBC lineages interact differently with the immune system potentially leading to distinct clinical manifestations (Tientcheu et al., 2017), and some genetic studies have been proof of concept that considering $M t b$ lineage in the analysis allows the finding of signals otherwise missed (Caws et al., 2008).

\section{MTB LINEAGE CHARACTERISTICS AND GEOLOCALIZATION}

The MTBC group, of which $M t b$ is part, comprises human- and animal-adapted species capable of causing disease in a wide range of hosts. The MTBC group consists of bacterial species with high DNA sequence similarity; however, genomic markers exist that allow classification of mycobacterial lineages (Brites and Gagneux, 2017). Within human-adapted strains, M. tuberculosis sensu stricto and M. africanum are divided in seven phylogenetic lineages, which cluster into preferred geographical locations. Mycobacterium tuberculosis strains are divided into lineages 1 to 4 , along with lineage 7 , while $M$. africanum strains fall into lineages 5 and 6 . In terms of geographical spread, lineages 5 to 7 are more restricted occurring mainly in West Africa (lineages 5 and 6) and Ethiopia (lineage 7). The distribution of lineages 1 and 3 is more diffuse, with lineage 1 mainly found in proximity to the Indian Ocean (hence also known as Indo-Oceanic strain), whereas lineage 3 is more commonly found in East Africa as well as Central and South Asia. The remaining lineage 2 (alias East Asia lineage) and lineage 4 (alias Euro-American lineage) are widely distributed globally. The Beijing family of $M t b$ strains are prominent members of lineage 2, whereas Harlem strains belong to lineage 4 . In addition, strains of lineages 2,3 , and 4 carry the genomic deletion TBD1 and are referred to as modern strains (Brites and Gagneux, 2017).

\section{EVIDENCE FOR MTB LINEAGE ASSOCIATION WITH HUMAN ETHNICITY}

The geographical distribution of lineages leads to a clear association with specific human populations (Gagneux et al., 2006). This sympatric pathogen-host relationship is reflective of comigration and coexpansion of human populations and $M t b$ (Comas et al., 2013; Luo et al., 2015; Stucki et al., 2016). The concept of $M t b$ and human host coadaptation gained additional support by a study of two Swiss cohorts. Subjects were followed for 8 and 10 years, and preferential infection by lineage 4 (EuroAmerican) was found in individuals born in Europe. Conversely, non-Europeans were more likely to be infected by other strains. The preferential host ethnicity- $M t b$ lineage relationship was maintained despite social mixing of subjects and after correction by age and sex (Fenner et al., 2013). These observations were consistent with other studies that also reported preferential associations of bacterial lineage with the phylogeographic region where TB patients were born even in areas with high pathogen and host ethnicity admixture (Hirsh et al., 2004; Reed et al., 2009; Pareek et al., 2013; Rasigade et al., 2017; Guthrie et al., 2018). For M. africanum, coadaptation with specific African subpopulations has been hypothesized as cause for its much restricted geographical distribution (Asante-Poku et al., 2015; Otchere et al., 2018); however, additional molecular epidemiology studies are needed. A major challenge in the interpretation of these results is the possibility of social contact bias, in which subjects from the same ethnicities tend to interact more frequently, hence increasing the chances for preferential ethnicity-lineage pairings. However, the case for true association is strengthened by the observation that preferential pairing of host ethnicity and $M t b$ lineage is not maintained in the case of HIV-TB coinfection, which occurs in the context of low CD4 T-cell counts (Fenner et al., 2013). Likewise, alterations in the transmission dynamics in defined geographical areas by introduction of foreign, more virulent strains can disrupt preferential pairings. For example, Beijing family strains (lineage 2) were shown to be overtransmitted in a Kinh Vietnamese population in contrast to lineage 1 . The increased virulence of Beijing strains in this setting was supported by shorter times of progression to active disease, wider geographical dispersal, and higher incidence of infection in younger individuals (Holt et al., 2018).

\section{THE INTERPLAY OF MTB LINEAGE AND THE HUMAN HOST}

It is known from murine studies that infection with different strains of Mtb results in significant differences in survival times for a given mouse strain. For instance, immunocompetent CB-17 mouse strains infected with Mtb HN878 (Lineage 2) resulted in increased mortality when contrasted against NHN5, HN60, CDC1551 and H37Rv strains (Manca et al., 2001). Likewise, when infecting different strains of inbred mice with the same Mtb strain, there are significant differences in survival times (Schurr and Kramnik, 2008). Variable disease outcomes promoted by $M t b$ lineages have also been demonstrated with common marmosets, an animal model that more closely resembles human TB. In contrast to mouse models, these animals develop lung pathology and infection with ancient (lineage 6 ) or modern lineages (2 and 4 ), resulting in different profiles of pulmonary damage, bacterial dissemination, and survival (Via et al., 2013). Distinct patterns of host responsiveness have also been observed for human 
studies. For example, a recent study assessed bacterial virulence of $M t b$ obtained from 153 Vietnamese TB patients employing a monocyte-derived macrophage lysis model. A high degree of macrophage lysis (i.e., high virulence) was associated with higher bacterial concentrations in sputum. High virulence strains also replicated faster in the macrophages and induced lower secretion of tumor necrosis factor $\alpha$ (TNF- $\alpha$ ) and IL-6, but higher production of IL-1 $\beta$. This high virulence bacterial phenotype was strongly associated with lineage 2 strains (Tram et al., 2018). However, this study did not address a possible "fit" between host and $M t b$ genetic background. The latter question was addressed by a human study, which analyzed cytokine production by monocyte-derived macrophages obtained from members of three ethnicities following infection with different strains of $M t b$. In univariate analyses, both Filipino ethnicity and $M t b$ strain CDC1551 were significant predictors of cytokine production. However, there was no evidence for significant interaction between ethnicity and $M t b$ strain possibly arguing against coadaptation in this sample (Nahid et al., 2018). Considering that a large number of tests were conducted (nine cytokines vs. four $M t b$ strains) in three groups with only 45 subjects per group, it is clear that power to detect such interactions was modest. Similar studies employing larger sample size and live bacterium challenges may provide different results.

A study of TB patients of Eurasian versus African ancestry (83 vs. 43 individuals) from London, UK, took a different approach. A large number of hematological parameters and soluble factors were determined at time of initiation of TB treatment, and five were found to be significantly different among the two ethnic groups. Similarly, whole blood was stimulated with $M t b$ antigens, soluble factors were determined, and IL-1RA and IL-12 concentrations were found significantly different between the two ethnicity groups (Coussens et al., 2013). However, in agreement with earlier studies, it was shown that these ethnicity-specific differences in immune responsiveness were independent of the lineage of the causative $M t b$ strain arguing against an interaction of strain and host genomes. In contrast, the observation of a strong genotypic impact of a VDBP polymorphisms on the response profile further supported the hypothesis that differences in immune responsiveness are primarily driven by host genetic variability (Coussens et al., 2013). While there was no significant impact of $M t b$ lineage by univariate analysis, it is possible that a significant interaction effect might have been detected; however, this was not tested. The genetic control for differences in immune response between European or African ancestry was investigated by genome-wide approaches, and extensive differences in inflammatory responses were detected by RNA sequencing (Nedelec et al., 2016). Along the line of unbiased genome-wide approaches, a detailed human-Mtb proteinprotein interaction map has recently been described (Penn et al., 2018). Unfortunately, $M t b$ lineage- or strain-specific data for the map are not yet available.

A common observation among studies was the reduced/ delayed triggering of a proinflammatory response upon infection of host cells with "modern lineages" (lineages 2-4) and a corresponding increased intracellular growth rate by lineages 2 and $4 \mathrm{Mtb}$ strains (reviewed in (Coscolla and Gagneux, 2014)).
However, strong heterogeneity was observed across studies. For example, Krishan et al. demonstrated a higher production of proinflammatory cytokines (i.e., TNF and IL1 $\beta$ ) in macrophages elicited by East Asian/Beijing and Indo-Oceanic strains in comparison to Euro-American lineages, whereas this modulation was less pronounced in dendritic cells (Krishnan et al., 2011) and absent from peripheral blood mononuclear cells (Portevin et al., 2011). By contrast, another study described low and high cytokine induction by lineages 1 and 2 in contrast to lineage 4, respectively (Reiling et al., 2013).

Differences in transmissibility of $M t b$ strains from patients to contacts have been noted repeatedly (Malik and Godfrey-Faussett, 2005). For example, lineages 2 and 4 are often referred to as the most transmissible strains, which could reflect the modulation of host immune response and hence increased virulence (Parwati et al., 2010). Indeed, a meta-analysis identified lineage 2 as the only lineage with a significantly increased risk of transmission employing lineage 4 strains as reference group (Wiens et al., 2018). However, there was substantial heterogeneity of results across studies. For example, lineage 2 was not overtransmitted in geographically diverse settings in Vietnam, The Gambia, Canada (Montreal and Alberta), and South Africa (Cape Town) (de Jong et al., 2008; Marais et al., 2009; Albanna et al., 2011; Buu et al., 2012; Langlois-Klassen et al., 2013). By contrast, lineage 3 (East African Indian strains) was undertransmitted in a Canadian study, even when analysis was conducted in patients where the strains are most frequently encountered (Albanna et al., 2011). This is in agreement with the results of the metaanalysis, which found lineage 3 strains presented reduced risk of transmission in Europe and Americas (Wiens et al., 2018). The same meta-analysis also found substantially increased risk of overtransmission of lineage 2 strains in East Asia (compared to other regions), whereas lineage 1 strains were associated with overtransmission only in East Asia (Wiens et al., 2018). It is not known if geographical differences in risk of transmission of certain $M t b$ strains reflect coadaptation of $M t b$ to specific human populations, other epidemiological or demographic factors, or the emergence of lineage substrains with greatly increased virulence and transmission potential (Rajwani et al., 2017).

Given that extrapulmonary TB cases present a lower risk for transmission of TB, we might expect preponderance of $\mathrm{PTB}$ as a result of coadaptation. Indeed, transmission potential has been linked to different pathways of pathogenesis (Verma et al., 2019). A study from Birmingham and Solihull, UK, reported increased extrathoracic TB for the East Asian lineage (lineage 2), but not for East African Indian lineage (lineage 1), using strains of the EuroAmerican lineage (lineage 4) as reference (Pareek et al., 2013). Similarly, Beijing strains (lineage 2) resulted in higher frequency of extrapulmonary TB compared to non-Beijing strains (Kong et al., 2007). By contrast, a retrospective study (2004-2015) in British Columbia, Canada, found that strains of the Indo-Oceanic lineage (lineage 1) were significantly enriched among cases with extrapulmonary TB compared with all other lineages (Guthrie et al., 2018). Consistent with the Canadian observations, a large US study of 32,000 TB cases with known $M t b$ lineage and disease site found that relative to all other lineages, lineage 2 (East Asian) was underrepresented among extrapulmonary TB cases. These 
results remained unchanged when adjusted for ethnicity or place of birth of the index case and collectively argued against a genetic interaction of $M t b$ and the human host in the determination of the clinical site of TB. However, given the conflicting results from different studies, it seems possible that lineage and ethnicity are too crude classifications and that a higher-resolution approach may be required.

Overall, these findings show a major impact of $M t b$ lineage on TB pathogenesis, which is a necessary factor for the coevolution of $M t b$ and its human host. Curiously, the strongest data in favor of human-Mtb coevolution arose in a host-free study. In a landmark study, Comas et al. (2010) found direct evidence for the action of selective pressures on the Mtb genome. Intriguingly, it was found that the strongest signs of selection were favoring conservation of T-cell epitopes across a large panel of Mtb strains (Comas et al., 2010). The most parsimonious explanation for this finding was that Mtb has adapted to human T-cell immunity and that aspects of the host T-cell response are beneficial for the transmission of Mtb (Comas et al., 2010; Sassetti and Rubin, 2010). However, much of the $M t b$ lineage impact on TB pathogenesis appears to transcend host genetics. For example, lineage 2 increases TB transmission and together with lineage 4 is more likely to cause severe pulmonary infection, irrespective of age, sex, and ethnicity (Rasigade et al., 2017; Wiens et al., 2018).

\section{EVIDENCE FOR HOST GENE/MTB GENOTYPE INTERACTION}

To address the problem of strain-specific genetic control in susceptibility to mycobacterial infection, a study employed recombinant congenic mice infected with BCG Russia and BCG Pasteur. In this design controlled for infection dosage and host genetic makeup, a strong linkage hit on chromosome 1 was identified for spleen-specific bacterial burden for both BCG Pasteur and BCG Russia infection. Interestingly, additional BCG Russia-specific linkage hits were detected on chromosomes 11 and 13 (Di Pietrantonio et al., 2010). By analyzing the host pathogen contribution to widely used readouts of host responsiveness to mycobacteria such as secretion of interferon $\gamma$, IL-4, IL-6, and IL-12, it was possible to detect significant interaction of host genetics and pathogen strain on cytokine production (Di Pietrantonio et al., 2011). These results established that under strictly controlled experimental conditions strain-specific differences in host susceptibility can be detected and that specificity exists in the host-mycobacteria interaction (Di Pietrantonio and Schurr, 2013).

In human populations, careful studies were able to demonstrate evidence for a human-Mtb genetic interplay, despite the experimental challenges such as disease heterogeneity among cases or low study power as consequence of sample size. These results are particularly encouraging, considering the intrinsic challenges of transmission studies and the complexity of TB pathogenesis. The first report for lineage-dependent SNV association with human TB was based on a case-control Vietnamese sample with mixed cases of PTB and TBM. In this work, contrasting controls to a subset of patients infected with the East Asian/Beijing lineage revealed the SNV T597C in TLR2 as a risk factor for TB. Further study of the lineage-dependent effect showed that the association was driven by the smaller subset of TBM patients infected with the Beijing genotype $\left(\mathrm{OR}=1.91, P=1 \times 10^{-3}\right.$ ) (Caws et al., 2008). Expanding the previous studied sample and applying a discovery and validation case-control design, two TLR9 SNVs in strong LD were found associated with a combined TB phenotype, with rs352142 displaying the most significant association signal $\left(\mathrm{OR}=2.33, P=4 \times 10^{-3}\right)$. To test for a lineage-specific effect, discovery and validation samples were combined, and rs352142 showed a trend of association with a subsample infected by $M t b$ belonging to the Indo-Oceanic or Euro-American lineages $\left(\chi^{2}=3.67, P=0.056\right)$ (Graustein et al., 2015). In Vietnamese subjects, SNV markers in the macrophage receptor MARCO were found associated with increased risk to PTB (rs2278589, OR = 1.6, $P=1 \times 10^{-3}$; rs6751745, $\mathrm{OR}=1.4$, $\left.P=9 \times 10^{-3}\right)$. Interestingly, a significant association was found between these two markers and the Beijing lineage, which was strongest under a heterozygous model $\left(\mathrm{OR}=1.7, P=1 \times 10^{-3}\right.$; and $\mathrm{OR}=1.5, P=1 \times 10^{-2}$, respectively) (Thuong et al., 2016). Testing of host genotypes with preferential association of $M t b$ lineage was conducted in two additional studies. An Indonesian study identified a strong association of SLC11A1 alleles with Beijing strains genotype, whereas a study of South Africans identified HLA class I alleles that were associated with strains of the Beijing group. While associations of host genotypes with $M t b$ lineage support the concept of host $M t b$ coadaptation, the reported results should be considered tentative until a better understanding of the implicated alleles with TB risk has been established (van Crevel et al., 2009; Salie et al., 2014).

In a Ghanaian sample, testing association of 18 polymorphisms in the IRGM gene with PTB resulted in no significant findings for the total sample. However, stratifying patients by $M t b$ or M. africanum/Mycobacterium bovis strain detected a protective association of rs9637876 (5' UTR in IRGM) against $M t b$ (OR = $\left.0.66, P=4.5 \times 10^{-3}\right)$. Further study revealed the protective effect of the IRGM variant to be significantly stronger for $M t b$ strains belonging to the Euro-American lineage (Intemann et al., 2009). Reinvestigating the Ghanaian case-control cohort revealed rs1800451 in MBL2 to be associated with protection from PTB caused by $M$. africanum but not by $M t b(\mathrm{OR}=0.6, P=0.008)$ (Thye et al., 2011). Following the findings for IRGM in West Africa, a study conducted in Indonesians failed to associate SNVs in IRGM and 13 additional autophagy-related genes with TB. Analysis stratified by patient $M t b$ isolate lineage reached borderline nominal significance for two genes, but remained non-significant after multiple-testing correction (Songane et al., 2012). To date, the only GWAS that took lineage into account was conducted in a combined set of two Thai population samples. Total sample screening failed to find SNVs associated with TB. However, stratified analysis based on $M t b$ lineage found association of rs $1418425\left(\mathrm{OR}=1.62, P=1.58 \times 10^{-7}\right.$, chromosome region 1p13, intergenic region of CD53 and LRIF1) in patients infected with non-Beijing lineage strains (61\% of patients, $49 \%$ being infected by East African Indian/Indo-Oceanic lineage). Further stratification based on non-Beijing-infected subjects 
older than 45 years yielded more significant results $(\mathrm{OR}=1.74$, $\left.P=2.54 \times 10^{-8}\right)($ Omae et al., 2017). Like the $M t b$ lineagedependent host genotype associations, these results need to be replicated in an independent sample to confirm the validity of the stratification approach.

\section{CONCLUSION}

While intuitively attractive and supported by conceptually consistent observations, strong data showing $M t b$-human coevolution are still missing. There are data that show how infectious pathogens have shaped the human genome with major impact on genes encoding proteins of the innate immune system. Yet, the identification of the specific pathogens that underlie selection of specific human variants has proven more difficult. Well-powered studies specifically designed to address the role of human-Mtb coadaptation are needed to follow up on the general concept, and such studies need to be complemented with specific examples of gene-gene interaction between the human host and $M t b$ isolates. From the human side, it may be interesting to focus on studies employing strong dominant acting $M t b$ resistance alleles, most likely to be found by studying $M t b$ infection, or strong recessive acting susceptibility alleles, such as provided by the TYK2 example. On the bacterial

\section{REFERENCES}

Abel, L., Fellay, J., Haas, D. W., Schurr, E., Srikrishna, G., Urbanowski, M., et al. (2018). Genetics of human susceptibility to active and latent tuberculosis: present knowledge and future perspectives. Lancet Infect. Dis. 18 (3), e64-e75. doi: 10.1016/S1473-3099(17)30623-0

Adams, L. A., Moller, M., Nebel, A., Schreiber, S., van der Merwe, L., van Helden, P. D., et al. (2011). Polymorphisms in MC3R promoter and CTSZ 3' UTR are associated with tuberculosis susceptibility. Eur. J. Hum. Genet. 19 (6), 676-681. doi: 10.1038/ejhg.2011.1

Albanna, A. S., Reed, M. B., Kotar, K. V., Fallow, A., McIntosh, F. A., Behr, M. A., et al. (2011). Reduced transmissibility of East African Indian strains of Mycobacterium tuberculosis. PLoS One 6 (9), e25075. doi: 10.1371/journal.pone.0025075

Alcais, A., Fieschi, C., Abel, L., and Casanova, J. L. (2005). Tuberculosis in children and adults: two distinct genetic diseases. J. Exp. Med. 202 (12), 1617-1621. doi: 10.1084/jem.20052302

Asante-Poku, A., Yeboah-Manu, D., Otchere, I. D., Aboagye, S. Y., Stucki, D., Hattendorf, J., et al. (2015). Mycobacterium africanum is associated with patient ethnicity in Ghana. PLoS Negl. Trop. Dis. 9 (1), e3370. doi: 10.1084/ jem.20060269

Baghdadi, J. E., Orlova, M., Alter, A., Ranque, B., Chentoufi, M., Lazrak, F., et al. (2006). An autosomal dominant major gene confers predisposition to pulmonary tuberculosis in adults. J. Exp. Med. 203 (7), 1679-1684. doi: 10.1084/jem.20060269

Barreiro, L. B., and Quintana-Murci, L. (2010). From evolutionary genetics to human immunology: how selection shapes host defence genes. Nat. Rev. Genet. 11 (1), 17-30. doi: 10.1038/nrg2698

Behr, M. A., Edelstein, P. H., and Ramakrishnan, L. (2018). Revisiting the timetable of tuberculosis. BMJ 362, k2738. doi: 10.1136/bmj.k2738

Boisson-Dupuis, S., Ramirez-Alejo, N., Li, Z., Patin, E., Rao, G., Kerner, G., et al. (2018). Tuberculosis and impaired IL-23-dependent IFN-gamma immunity in humans homozygous for a common TYK2 missense variant. Sci. Immunol. 3 (30), 1-30. doi: 10.1126/sciimmunol.aau8714

Brites, D., and Gagneux, S. (2015). Co-evolution of Mycobacterium tuberculosis and Homo sapiens. Immunol. Rev. 264 (1), 6-24. doi: 10.1111/imr.12264 side, a follow-up on protective epitopes stratified by human hosts may be equally informative. In general terms, it seems likely that the stratification by bacterial linages is too crude to allow significant conclusions. Future studies likely will need to consider high-resolution genomic substrains (Coll et al., 2014; Stucki et al., 2016), which, unfortunately, will increase the required sample size. While the challenges are daunting, they may well be worth the effort. The study of biological problems and their relevance for human health has provided numerous examples that ignoring the powerful forces of evolution may not be a wise choice.

\section{AUTHOR CONTRIBUTIONS}

WC-M and GC contributed equally to the literature review and manuscript writing. ES contributed to the writing of the manuscript. All authors read and approved the final manuscript.

\section{FUNDING}

Research in the authors' laboratory is supported by a foundation grant from the Canadian Institute of Health Research (grant 143332).

Brites, D., and Gagneux, S. (2017). The nature and evolution of genomic diversity in the Mycobacterium tuberculosis complex. Adv. Exp. Med. Biol. 1019, 1-26. doi: 10.1007/978-3-319-64371-7_1

Buu, T. N., van Soolingen, D., Huyen, M. N., Lan, N. T., Quy, H. T., Tiemersma, E. W., et al. (2012). Increased transmission of Mycobacterium tuberculosis Beijing genotype strains associated with resistance to streptomycin: a populationbased study. PLoS One 7 (8), e42323. doi: 10.1371/journal.pone.0042323

Caws, M., Thwaites, G., Dunstan, S., Hawn, T. R., Lan, N. T., Thuong, N. T., et al. (2008). The influence of host and bacterial genotype on the development of disseminated disease with Mycobacterium tuberculosis. PLoS Pathog. 4 (3), e1000034. doi: 10.1371/journal.ppat.1000034

Chimusa, E. R., Zaitlen, N., Daya, M., Moller, M., van Helden, P. D., Mulder, N. J., et al. (2014). Genome-wide association study of ancestry-specific TB risk in the South African Coloured population. Hum. Mol. Genet. 23 (3), 796-809. doi: $10.1093 / \mathrm{hmg} / \mathrm{ddt} 462$

Cobat, A., Gallant, C. J., Simkin, L., Black, G. F., Stanley, K., Hughes, J., et al. (2009). Two loci control tuberculin skin test reactivity in an area hyperendemic for tuberculosis. J. Exp. Med. 206 (12), 2583-2591. doi: 10.1084/jem.20090892

Cobat, A., Poirier, C., Hoal, E., Boland-Auge, A., de La Rocque, F., Corrard, F., et al. (2015). Tuberculin skin test negativity is under tight genetic control of chromosomal region 11p14-15 in settings with different tuberculosis endemicities. J. Infect. Dis. 211 (2), 317-321. doi: 10.1093/infdis/jiu446

Coll, F., McNerney, R., Guerra-Assuncao, J. A., Glynn, J. R., Perdigao, J., Viveiros, M., et al. (2014). A robust SNP barcode for typing Mycobacterium tuberculosis complex strains. Nat. Commun. 5, 4812. doi: 10.1038/ncomms5812

Comas, I., Chakravartti, J., Small, P. M., Galagan, J., Niemann, S., Kremer, K., et al. (2010). Human T cell epitopes of Mycobacterium tuberculosis are evolutionarily hyperconserved. Nat. Genet. 42 (6), 498-503. doi: 10.1038/ng.590

Comas, I., Coscolla, M., Luo, T., Borrell, S., Holt, K. E., Kato-Maeda, M., et al. (2013). Out-of-Africa migration and neolithic coexpansion of Mycobacterium tuberculosis with modern humans. Nat. Genet. 45 (10), 1176-1182. doi: 10.1038/ng.2744

Cooke, G. S., Campbell, S. J., Bennett, S., Lienhardt, C., McAdam, K. P., Sirugo, G., et al. (2008). Mapping of a novel susceptibility locus suggests a role for MC3R and CTSZ in human tuberculosis. Am. J. Respir. Crit. Care Med. 178 (2), $203-$ 207. doi: $10.1164 /$ rccm.200710-1554OC 
Coscolla, M., and Gagneux, S. (2014). Consequences of genomic diversity in Mycobacterium tuberculosis. Semin. Immunol. 26 (6), 431-444. doi: 10.1016/j. smim.2014.09.012

Coussens, A. K., Wilkinson, R. J., Nikolayevskyy, V., Elkington, P. T., Hanifa, Y., Islam, K., et al. (2013). Ethnic variation in inflammatory profile in tuberculosis. PLoS Pathog. 9 (7), e1003468. doi: 10.1371/journal.ppat.1003468

Dallmann-Sauer, M., Correa-Macedo, W., and Schurr, E. (2018). Human genetics of mycobacterial disease. Mamm. Genome 29 (7-8), 523-538. doi: 10.1007/ s00335-018-9765-4

de Jong, B. C., Hill, P. C., Aiken, A., Awine, T., Antonio, M., Adetifa, I. M., et al. (2008). Progression to active tuberculosis, but not transmission, varies by Mycobacterium tuberculosis lineage in the Gambia. J. Infect. Dis. 198 (7), 10371043. doi: 10.1086/591504

Di Pietrantonio, T., Correa, J. A., Orlova, M., Behr, M. A., and Schurr, E. (2011). Joint effects of host genetic background and mycobacterial pathogen on susceptibility to infection. Infect. Immun. 79 (6), 2372-2378. doi: 10.1128/ IAI.00985-10

Di Pietrantonio, T., Hernandez, C., Girard, M., Verville, A., Orlova, M., Belley, A., et al. (2010). Strain-specific differences in the genetic control of two closely related mycobacteria. PLoS Pathog. 6 (10), e1001169. doi: 10.1371/journal. ppat.1001169

Di Pietrantonio, T., and Schurr, E. (2013). Host-pathogen specificity in tuberculosis. Adv. Exp. Med. Biol. 783, 33-44. doi: 10.1007/978-1-4614-6111-1_2

Fenner, L., Egger, M., Bodmer, T., Furrer, H., Ballif, M., Battegay, M., et al. (2013). HIV infection disrupts the sympatric host-pathogen relationship in human tuberculosis. PLoS Genet. 9 (3), e1003318. doi: 10.1371/journal.pgen.1003318

Fox, G. J., Orlova, M., and Schurr, E. (2016). Tuberculosis in newborns: the lessons of the "Lubeck disaster" (1929-1933). PLoS Pathog. 12 (1), e1005271. doi: 10.1371/journal.ppat.1005271

Gagneux, S., DeRiemer, K., Van, T., Kato-Maeda, M., de Jong, B. C., Narayanan, S., et al. (2006). Variable host-pathogen compatibility in Mycobacterium tuberculosis. Proc. Natl. Acad. Sci. U.S.A. 103 (8), 2869-2873. doi: 10.1073/pnas.0511240103

Grant, A. V., El Baghdadi, J., Sabri, A., El Azbaoui, S., Alaoui-Tahiri, K., Abderrahmani Rhorfi, I., et al. (2013). Age-dependent association between pulmonary tuberculosis and common TOX variants in the 8q12-13 linkage region. Am. J. Hum. Genet. 92 (3), 407-414. doi: 10.1016/j.ajhg.2013.01.013

Grant, A. V., Sabri, A., Abid, A., Abderrahmani Rhorfi, I., Benkirane, M., Souhi, H., et al. (2016). A genome-wide association study of pulmonary tuberculosis in Morocco. Hum. Genet. 135 (3), 299-307. doi: 10.1007/s00439-016-1633-2

Graustein, A. D., Horne, D. J., Arentz, M., Bang, N. D., Chau, T. T., Thwaites, G. E., et al. (2015). TLR9 gene region polymorphisms and susceptibility to tuberculosis in Vietnam. Tuberculosis (Edinb) 95 (2), 190-196. doi: 10.1016/j. tube.2014.12.009

Guthrie, J. L., Kong, C., Roth, D., Jorgensen, D., Rodrigues, M., Hoang, L., et al. (2018). Molecular epidemiology of tuberculosis in British Columbia, Canada: a 10-year retrospective study. Clin. Infect. Dis. 66 (6), 849-856. doi: 10.1093/ $\mathrm{cid} / \mathrm{cix} 906$

Hashemi, M., Eskandari-Nasab, E., Moazeni-Roodi, A., Naderi, M., SharifiMood, B., and Taheri, M. (2013). Association of CTSZ rs34069356 and MC3R rs6127698 gene polymorphisms with pulmonary tuberculosis. Int. J. Tuberc. Lung Dis. 17 (9), 1224-1228. doi: 10.5588/ijtld.12.0762

Hirsh, A. E., Tsolaki, A. G., DeRiemer, K., Feldman, M. W., and Small, P. M. (2004). Stable association between strains of Mycobacterium tuberculosis and their human host populations. Proc. Natl. Acad. Sci. U.S.A. 101 (14), 4871-4876. doi: 10.1073/pnas.0305627101

Holt, K. E., McAdam, P., Thai, P. V. K., Thuong, N. T. T., Ha, D. T. M., Lan, N. N., et al. (2018). Frequent transmission of the Mycobacterium tuberculosis Beijing lineage and positive selection for the EsxW Beijing variant in Vietnam. Nat. Genet. 50 (6), 849-856. doi: 10.1038/s41588-018-0117-9

Intemann, C. D., Thye, T., Niemann, S., Browne, E. N., Amanua Chinbuah, M., Enimil, A., et al. (2009). Autophagy gene variant IRGM -261T contributes to protection from tuberculosis caused by Mycobacterium tuberculosis but not by $M$. africanum strains. PLoS Pathog. 5 (9), e1000577. doi: 10.1371/journal.ppat.1000577

Kallmann, F. J., and Reisner, D. (1943). Twin studies on the significance of genetic factors in tuberculosis. Am. Rev. Respir. Dis. 47, 549-574. doi: 10.1093/ oxfordjournals.jhered.a105302

Kerner, G., Ramirez-Alejo, N., Seeleuthner, Y., Yang, R., Ogishi, M., Cobat, A., et al. (2019). Homozygosity for TYK2 P1104A underlies tuberculosis in about
$1 \%$ of patients in a cohort of European ancestry. Proc. Natl. Acad. Sci. U.S.A. 116 (21), 10430-10434. doi: 10.1073/pnas.1903561116

Kodaman, N., Pazos, A., Schneider, B. G., Piazuelo, M. B., Mera, R., Sobota, R. S., et al. (2014). Human and Helicobacter pylori coevolution shapes the risk of gastric disease. Proc. Natl. Acad. Sci. U.S.A. 111 (4), 1455-1460. doi: 10.1073/ pnas. 1318093111

Kong, Y., Cave, M. D., Zhang, L., Foxman, B., Marrs, C. F., Bates, J. H., et al. (2007). Association between Mycobacterium tuberculosis Beijing/W lineage strain infection and extrathoracic tuberculosis: insights from epidemiologic and clinical characterization of the three principal genetic groups of $M$. tuberculosis clinical isolates. J. Clin. Microbiol. 45 (2), 409-414. doi: 10.1128/JCM.01459-06

Krishnan, N., Malaga, W., Constant, P., Caws, M., Tran, T. H., Salmons, J., et al. (2011). Mycobacterium tuberculosis lineage influences innate immune response and virulence and is associated with distinct cell envelope lipid profiles. PLoS One 6 (9), e23870. doi: 10.1371/journal.pone.0023870

Langlois-Klassen, D., Senthilselvan, A., Chui, L., Kunimoto, D., Saunders, L. D., Menzies, D., et al. (2013). Transmission of Mycobacterium tuberculosis Beijing Strains, Alberta, Canada, 1991-2007. Emerg. Infect. Dis. 19 (5), 701-711. doi: 10.3201/eid1905.121578

Luo, T., Comas, I., Luo, D., Lu, B., Wu, J., Wei, L., et al. (2015). Southern East Asian origin and coexpansion of Mycobacterium tuberculosis Beijing family with Han Chinese. Proc. Natl. Acad. Sci. U. S. A. 112 (26), 8136-8141. doi: 10.1073/ pnas. 1424063112

Mahasirimongkol, S., Yanai, H., Nishida, N., Ridruechai, C., Matsushita, I., Ohashi, J., et al. (2009). Genome-wide SNP-based linkage analysis of tuberculosis in Thais. Genes Immun. 10 (1), 77-83. doi: 10.1038/gene.2008.81

Malik, A. N., and Godfrey-Faussett, P. (2005). Effects of genetic variability of Mycobacterium tuberculosis strains on the presentation of disease. Lancet Infect. Dis. 5 (3), 174-183. doi: 10.1016/S1473-3099(05)01310-1

Manca, C., Tsenova, L., Bergtold, A., Freeman, S., Tovey, M., Musser, J. M., et al. (2001). Virulence of a Mycobacterium tuberculosis clinical isolate in mice is determined by failure to induce $\mathrm{T}_{\mathrm{H}} 1$ type immunity and is associated with induction of IFN-alpha/beta. Proc. Natl. Acad. Sci. U. S. A. 98 (10), 5752-5757. doi: 10.1073/pnas.091096998

Marais, B. J., Hesseling, A. C., Schaaf, H. S., Gie, R. P., van Helden, P. D., and Warren, R. M. (2009). Mycobacterium tuberculosis transmission is not related to household genotype in a setting of high endemicity. J. Clin. Microbiol. 47 (5), 1338-1343. doi: 10.1128/JCM.02490-08

Nahid, P., Jarlsberg, L. G., Kato-Maeda, M., Segal, M. R., Osmond, D. H., Gagneux, S., et al. (2018). Interplay of strain and race/ethnicity in the innate immune response to M. tuberculosis. PLoS One 13 (5), e0195392. doi: 10.1371/ journal.pone.0195392

Nedelec, Y., Sanz, J., Baharian, G., Szpiech, Z. A., Pacis, A., Dumaine, A., et al. (2016). Genetic ancestry and natural selection drive population differences in immune responses to pathogens. Cell 167 (3), 657-669.e621. doi: 10.1016/j. cell.2016.09.025

Omae, Y., Toyo-Oka, L., Yanai, H., Nedsuwan, S., Wattanapokayakit, S., Satproedprai, N., et al. (2017). Pathogen lineage-based genome-wide association study identified CD53 as susceptible locus in tuberculosis. J. Hum. Genet. 62 (12), 1015-1022. doi: 10.1038/jhg.2017.82

Orlova, M., and Schurr, E. (2017). Human genomics of Mycobacterium tuberculosis infection and disease. Curr. Genet. Med. Rep. 5 (3), 125-131. doi: 10.1007/ s40142-017-0124-7

Otchere, I. D., Coscolla, M., Sanchez-Buso, L., Asante-Poku, A., Brites, D., Loiseau, C., et al. (2018). Comparative genomics of Mycobacterium africanum lineage 5 and lineage 6 from Ghana suggests distinct ecological niches. Sci. Rep. 8 (1), 11269. doi: 10.1038/s41598-018-29620-2

Pareek, M., Evans, J., Innes, J., Smith, G., Hingley-Wilson, S., Lougheed, K. E., et al. (2013). Ethnicity and mycobacterial lineage as determinants of tuberculosis disease phenotype. Thorax 68 (3), 221-229. doi: 10.1136/thoraxjnl-2012-201824

Parwati, I., van Crevel, R., and van Soolingen, D. (2010). Possible underlying mechanisms for successful emergence of the Mycobacterium tuberculosis Beijing genotype strains. Lancet Infect. Dis. 10 (2), 103-111. doi: 10.1016/ S1473-3099(09)70330-5

Paulson, T. (2013). Epidemiology: a mortal foe. Nature 502 (7470), S2-S3. doi: $10.1038 / 502 S 2 a$

Penn, B. H., Netter, Z., Johnson, J. R., Von Dollen, J., Jang, G. M., Johnson, T., et al. (2018). An $M t b$-human protein-protein interaction map identifies a switch 
between host antiviral and antibacterial responses. Mol. Cell. 71 (4), 637-648. e635. doi: 10.1016/j.molcel.2018.07.010

Portevin, D., Gagneux, S., Comas, I., and Young, D. (2011). Human macrophage responses to clinical isolates from the Mycobacterium tuberculosis complex discriminate between ancient and modern lineages. PLoS Pathog. 7 (3), e1001307. doi: 10.1371/journal.ppat.1001307

Puffer, R. R. (1944). Familial susceptibility to tuberculosis: its importance as a public health problem. Cambridge, MA: Harvard Univ. Press, 106 pp. doi: 10.4159/ harvard. 9780674428720

Quintana-Murci, L. (2019). Human immunology through the lens of evolutionary genetics. Cell 177 (1), 184-199. doi: 10.1016/j.cell.2019.02.033

Rajwani, R., Yam, W. C., Zhang, Y., Kang, Y., Wong, B. K. C., Leung, K. S. S., et al. (2017). Comparative whole-genomic analysis of an ancient 12 Lineage Mycobacterium tuberculosis reveals a novel phylogenetic clade and common genetic determinants of hypervirulent strains. Front. Cell. Infect. Microbiol. 7, 539. doi: 10.3389/fcimb.2017.00539

Rasigade, J.P., Barbier,M.,Dumitrescu, O., Pichat, C., Carret, G., Ronnaux-Baron, A.S., et al. (2017). Strain-specific estimation of epidemic success provides insights into the transmission dynamics of tuberculosis. Sci. Rep. 7, 45326. doi: 10.1038/ srep45326

Reed, M. B., Pichler, V. K., McIntosh, F., Mattia, A., Fallow, A., Masala, S., et al. (2009). Major Mycobacterium tuberculosis lineages associate with patient country of origin. J. Clin. Microbiol. 47 (4), 1119-1128. doi: 10.1128/JCM.02142-08

Reiling, N., Homolka, S., Walter, K., Brandenburg, J., Niwinski, L., Ernst, M., et al. (2013). Clade-specific virulence patterns of Mycobacterium tuberculosis complex strains in human primary macrophages and aerogenically infected mice. $M B i .4$ (4), 1-10. doi: 10.1128/mBio.00250-13

Salie, M., van der Merwe, L., Moller, M., Daya, M., van der Spuy, G. D., van Helden, P. D., et al. (2014). Associations between human leukocyte antigen class I variants and the Mycobacterium tuberculosis subtypes causing disease. J. Infect. Dis. 209 (2), 216-223. doi: 10.1093/infdis/jit443

Sassetti, C. M., and Rubin, E. J. (2010). Relics of selection in the mycobacterial genome. Nat. Genet. 42 (6), 476-478. doi: 10.1038/ng0610-476

Schurr, E., and Kramnik, I. (2008). "Genetic control of host susceptibility to tuberculosis," in Handbook of tuberculosis. Eds. S. H. E. Kaufmann and W. J. Britten (Weinheim, Germany: Wiley-VCH).

Simmons, J. D., Stein, C. M., Seshadri, C., Campo, M., Alter, G., Fortune, S., et al. (2018). Immunological mechanisms of human resistance to persistent Mycobacterium tuberculosis infection. Nat. Rev. Immunol. 18 (9), 575-589. doi: 10.1038/s41577-018-0025-3

Sobota, R. S., Stein, C. M., Kodaman, N., Scheinfeldt, L. B., Maro, I., WielandAlter, W., et al. (2016). A locus at 5q33.3 confers resistance to tuberculosis in highly susceptible individuals. Am. J. Hum. Genet. 98 (3), 514-524. doi: 10.1016/j.ajhg.2016.01.015

Songane, M., Kleinnijenhuis, J., Alisjahbana, B., Sahiratmadja, E., Parwati, I., Oosting, M., et al. (2012). Polymorphisms in autophagy genes and susceptibility to tuberculosis. PLoS One 7 (8), e41618. doi: 10.1371/journal.pone.0041618

Stein, C. M., Sausville, L., Wejse, C., Sobota, R. S., Zetola, N. M., Hill, P. C., et al. (2017). Genomics of human pulmonary tuberculosis: from genes to pathways. Curr. Genet. Med. Rep. 5 (4), 149-166. doi: 10.1007/s40142-017-0130-9

Stein, C. M., Zalwango, S., Malone, L. L., Won, S., Mayanja-Kizza, H., Mugerwa, R. D., et al. (2008). Genome scan of $M$. tuberculosis infection and disease in Ugandans. PLoS One 3 (12), e4094. doi: 10.1371/journal.pone.0004094

Stucki, D., Brites, D., Jeljeli, L., Coscolla, M., Liu, Q., Trauner, A., et al. (2016). Mycobacterium tuberculosis lineage 4 comprises globally distributed and geographically restricted sublineages. Nat. Genet. 48 (12), 1535-1543. doi: 10.1038/ng.3704

Sveinbjornsson, G., Gudbjartsson, D. F., Halldorsson, B. V., Kristinsson, K. G., Gottfredsson, M., Barrett, J. C., et al. (2016). HLA class II sequence variants influence tuberculosis risk in populations of European ancestry. Nat. Genet. 48 (3), 318-322. doi: 10.1038/ng.3498
Thuong, N. T., Tram, T. T., Dinh, T. D., Thai, P. V., Heemskerk, D., Bang, N. D., et al. (2016). MARCO variants are associated with phagocytosis, pulmonary tuberculosis susceptibility and Beijing lineage. Genes Immun. 17 (7), 419-425. doi: 10.1038 /gene.2016.43

Thwaites, G. E., van Toorn, R., and Schoeman, J. (2013). Tuberculous meningitis: more questions, still too few answers. Lancet Neurol. 12 (10), 999-1010. doi: 10.1016/S1474-4422(13)70168-6

Thye, T., Browne, E. N., Chinbuah, M. A., Gyapong, J., Osei, I., Owusu-Dabo, E., et al. (2009). IL10 haplotype associated with tuberculin skin test response but not with pulmonary TB. PLoS One 4 (5), e5420. doi: 10.1371/journal. pone. 0005420

Thye, T., Niemann, S., Walter, K., Homolka, S., Intemann, C. D., Chinbuah, M. A., et al. (2011). Variant G57E of mannose binding lectin associated with protection against tuberculosis caused by Mycobacterium africanum but not by M. tuberculosis. PLoS One 6 (6), e20908. doi: 10.1371/journal.pone.0020908

Thye, T., Owusu-Dabo, E., Vannberg, F. O., van Crevel, R., Curtis, J., Sahiratmadja, E., et al. (2012). Common variants at $11 \mathrm{p} 13$ are associated with susceptibility to tuberculosis. Nat. Genet. 44 (3), 257-259. doi: 10.1038/ng.1080

Tientcheu, L. D., Koch, A., Ndengane, M., Andoseh, G., Kampmann, B., and Wilkinson, R. J. (2017). Immunological consequences of strain variation within the Mycobacterium tuberculosis complex. Eur. J. Immunol. 47 (3), 432-445. doi: 10.1002/eji.201646562

Tram, T. T. B., Nhung, H. N., Vijay, S., Hai, H. T., Thu, D. D. A., Ha, V. T. N., et al. (2018). Virulence of Mycobacterium tuberculosis clinical isolates is associated with sputum pre-treatment bacterial load, lineage, survival in macrophages, and cytokine response. Front. Cell. Infect. Microbiol. 8, 417. doi: 10.3389/ fcimb.2018.00417

van Crevel, R., Parwati, I., Sahiratmadja, E., Marzuki, S., Ottenhoff, T. H., Netea, M. G., et al. (2009). Infection with Mycobacterium tuberculosis Beijing genotype strains is associated with polymorphisms in SLC11A1/NRAMP1 in Indonesian patients with tuberculosis. J. Infect. Dis. 200 (11), 1671-1674. doi: $10.1086 / 648477$

Verma, S., Bhatt, K., Lovey, A., Ribeiro-Rodrigues, R., Durbin, J., Jones-López, E. C., et al. (2019). Transmission phenotype of Mycobacterium tuberculosis strains is mechanistically linked to induction of distinct pulmonary pathology. PLoS Pathog. 15 (3), e1007613. doi: 10.1371/journal.ppat.1007613

Via, L. E., Weiner, D. M., Schimel, D., Lin, P. L., Dayao, E., Tankersley, S. L., et al. (2013). Differential virulence and disease progression following Mycobacterium tuberculosis complex infection of the common marmoset (Callithrix jacchus). Infect. Immun. 81 (8), 2909-2919. doi: 10.1128/IAI.00632-13

Wiens, K. E., Woyczynski, L. P., Ledesma, J. R., Ross, J. M., Zenteno-Cuevas, R., Goodridge, A., et al. (2018). Global variation in bacterial strains that cause tuberculosis disease: a systematic review and meta-analysis. BMC Med. 16 (1), 196. doi: 10.1186/s12916-018-1180-x

World Health Organization (2018). Global tuberculosis report 2018. Geneva: World Health Organization. http://www.who.int/iris/handle/10665/274453. License: CC BY-NC-SA 3.0 IGO,

Zumla, A., Raviglione, M., Hafner, R., and von Reyn, C. F. (2013). Tuberculosis. N. Engl. J. Med. 368 (8), 745-755. doi: 10.1056/NEJMra1200894

Conflict of Interest Statement: The authors declare that the research was conducted in the absence of any commercial or financial relationships that could be construed as a potential conflict of interest.

Copyright $\odot 2019$ Correa-Macedo, Cambri and Schurr. This is an open-access article distributed under the terms of the Creative Commons Attribution License (CC BY). The use, distribution or reproduction in other forums is permitted, provided the original author(s) and the copyright owner(s) are credited and that the original publication in this journal is cited, in accordance with accepted academic practice. No use, distribution or reproduction is permitted which does not comply with these terms. 